\title{
Prácticas de Gestión de Relaciones con Clientes (CRM) en pequeñas empresas, periodo 2010 al 2019
}

\section{Customer Relations Management (CRM) practices in small business, period 2010 to 2019}

\section{RESUMEN}

El propósito de este estudio es determinar el nivel de interés por investigaciones relacionadas a prácticas de Gestión de Relaciones con Clientes (CRM) en pequeñas empresas del 2010 al 2019, en las principales revistas científicas de marketing. La metodología empleada consiste en la revisión de literatura sobre CRM en una muestra de 151 artículos publicados en la última década de las principales revistas de marketing, ubicadas en el primer cuartil Q1, según Scimago Journal Rank. Los resultados demuestran que de 125 artículos empíricos dedicados a la Gestión de Relaciones con Clientes (CRM), 72 publicaciones obtienen información de la gran empresa, seguido de 46 artículos que extraen simultáneamente datos de la pequeña, mediana y gran empresa; y solo 7 artículos de CRM reportan la realidad de las pequeñas empresas. Se concluye que hay mayor interés por desarrollar investigaciones de CRM dirigido hacia las grandes corporaciones, en comparación con las pequeñas empresas.
\end{abstract}

Palabras claves: Marketing Relacional; Gestión de Relaciones con Clientes (CRM); pequeñas empresas; revisión de la literatura. \begin{abstract}
The purpose of this study is to determine the level of interest in research related to Customer Relationship Management (CRM) practices in small companies from 2010 to 2019, in the main marketing scientific journals. The methodology used consists of the literature review on CRM in a sample of 151 articles published in the last decade of the main marketing journals, located in the first quartile Q1, according to Scimago Journal Rank. The results show that out of 125 empirical articles devoted to Customer Relationship Management (CRM), 72 publications obtain information from large companies, followed by 46 articles that simultaneously extract data from small, medium and large companies; and only 7 CRM articles report
\end{abstract}

Johana Elizabeth Arango Aramburu $^{1}$

johana.arango@unmsm.edu.pe ORCID: https://orcid.org/00000002-6559-2321

Mauro Amaru Granados Maguiño ${ }^{1}$ mgranadosm@unmsm.edu.pe ORCID: https://orcid.org/00000002-5668-0557

${ }^{1}$ Universidad Nacional Mayor de San Marcos, Facultad de Ciencias Administrativas. Lima, Perú

Presentado: 09/02/2021 - Aceptado: 18/03/2021 - Publicado: 17/06/2021

(C) Los autores. Este artículo es publicado por la revista Gestión en el Tercer Milenio de la Facultad de Ciencias Administrativas Universidad Nacional Mayor de San Marcos. Este es un artículo de acceso abierto, distribuido bajo los términos de la licencia Creative Commons Atribución 4.0 Internacional (CC BY 4.0) [https://creativecommons.org/licenses/by/4.0/deed.es] que permite el uso, distribución y reproducción en cualquier medio, siempre que la obra original sea debidamente citada de su fuente original. 
the reality of small businesses. It is concluded that there is greater interest in developing CRM research directed towards large corporations, compared to small companies.

Keywords: Relationship Marketing; Customer Relationship Management (CRM); small companies; review of the literature.

\section{INTRODUCCIÓN}

El objetivo fundamental de este trabajo es analizar el nivel de producción científica sobre Gestión de Relaciones con Clientes o Customer Relationship Management (CRM) en pequeñas empresas del 2010 al 2019.

En tal sentido, ante una realidad altamente digital, cambiante y competitiva, hoy las organizaciones buscan el crecimiento sostenible y la máxima rentabilidad a partir de la fidelización de clientes de valor; este reto se hace más complejo en pequeñas empresas agobiadas por estructuras y procesos organizacionales lentos y verticales.

Asimismo, desde el campo del marketing se alienta a las organizaciones a generar un esfuerzo para optimizar la interacción comercial con clientes rentables a través del Marketing Relacional, el cual busca mantener e intensificar relaciones beneficiosas entre las partes para lograr posteriormente la satisfacción y lealtad. Este proceso se inicia con la diferenciación de los productos y servicios a través de una propuesta de valor alimentado por el acceso de información valiosa del cliente. Es por ello que una de las herramientas del Marketing Relacional que facilita este trabajo es la Gestión de Relaciones con Clientes (CRM) la cual permite interacciones comunicativas sólidas y continuas a partir de la oferta de productos y servicios adaptado a las necesidades de cada cliente.

Al respecto, en los últimos 30 años, la literatura sobre la conceptualización del CRM ha sido variada y, a la vez, muy fragmentada. Sin embargo, se destaca en este trabajo la perspectiva de investigadores que reconocen que el CRM debe ser entendido como estrategia de negocio a largo plazo donde lo más valioso es la interacción con los clientes. Así lo señalan Zikmund, McLeod y Gilbert (2004) quienes afirman que:

El CRM es una estrategia de negocio que utiliza la tecnología de la información para que la organización tenga un panorama total e integrado de cada segmento; de esta forma los procesos y las interacciones con los clientes puedan permitir una relación beneficiosa para las partes. (p. 3)

Este aporte evidencia la importancia de la gestión de la información a través de la tecnología como elemento intangible valioso que permite moldear formas de interacción y ayuda mutua proveedor-clientes.

En esta línea, Pan y Lee definen la Gestión de Relaciones con el Cliente (CRM) como "una estrategia de negocio que se desarrolla mediante el trabajo interfuncional de diversas áreas de atención al cliente a través de la eficacia en la gestión de personas, procesos y tecnología" (2003, p. 96). En esta cita se destaca las habilidades gerenciales para la buena gestión del equipo colaborador de la organización, así como un conjunto de fases interrelacionadas y retroalimentadas por los flujos de información que brinda los recursos tecnológicos.

Por su parte, Finnegan y Currie (2010) señalan que:

El CRM no sólo está relacionado a tecnología o software, sino que lo importante es entenderlo como un enfoque estratégico integral que permite gestionar la evolución de las relaciones con los clientes para dar respuesta a las necesidades cambiantes de los mercados. (p. 155)

En el presente estudio, se conceptualiza CRM como un conjunto de estrategias interfuncionales a distintos niveles organizacionales que permiten optimizar las relaciones con cada cliente a fin de lograr una ventaja diferencial para competir en el mercado.

$\mathrm{Si}$ bien en las definiciones citadas de CRM se destaca las buenas relaciones comprador-vendedor, sin embargo, para el éxito en la 
implementación de programas CRM se necesita la puesta en marcha de múltiples elementos. Smith et al., 1999; Britton y Rose, 2004; Fang et al., 2011, citado en Nguyen y Mutum D. (2012) señalan "la confianza, el compromiso, satisfacción, simetría, dependencia y equidad entre la empresa y los clientes como pilares fundamentales en el proceso" (p. 8).

Por otra parte, Dalla, Goetz y Sahut (2018) refieren que "el éxito de todo proceso de CRM está en la implementación integral en un determinado periodo de tiempo de ciertos elementos claves de CRM como alineación organizacional, estrategia, tecnología CRM y Gestión de Clientes" (p. 392).

Asimismo, los estudios relacionados a la revisión de la literatura del CRM han demostrado significativos avances en la comprensión de tendencias y área temáticas de interés en las organizaciones. El más reciente es el de Sota, Chaudhry, Chamaria y Chauhan (2018) que analizó entre otros criterios, los temas clave del CRM del 2007 al 2016; su estudio demostró que "el tema más investigado son los relacionados a programas de lealtad y recompensa en grandes empresas, seguido del uso del CRM con enfoque transcultural" (p. 5).

Por su parte, Wahlberg, Strandberg, Sundberg y Sandberg (2009) en el estudio denominado tendencias, temas y áreas poco investigadas en la investigación de CRM: una revisión de la literatura, concluyó que la mayor parte de los artículos revisados entre 1988 al 2006 son de tipo CRM estratégico el cual visiona las formas de intervención a largo plazo y CRM analítico que "prioriza la información del cliente almacenada en base de datos; en ambos casos el estudio analizó la realidad de grandes empresas que operan en ámbitos geográficos extensos dejando claro que faltan estudios de empresas a pequeña escala" (p. 204).

Ngai (2005) en la investigación sobre la Gestión de Relaciones con los Clientes, periodo 1992-2002: revisión y clasificación de la literatura académica, logró descubrir las áreas temáticas de creciente interés en el tiempo. "Por un lado lo concerniente a planificación y estrategias CRM y; por otro lado, reportó una larga lista de artículos relacionados a software, minería de datos, gestión del conocimiento y comercio electrónico" (Ngai, 2005, p. 601).

\section{MÉTODOS}

En la presente investigación de revisión de la literatura se seleccionó a las cinco principales revistas de marketing del mundo registradas en el primer cuartil Q1 que reciben la mayor influencia científica a través del número de citas ponderadas, indicador SJR de factor de mayor impacto, según reporte de SCImago Journal Rank y la base de datos Scopus. Ellas son Marketing Journal of Marketing, Journal of the Academy Marketing Science, Journal of Marketing Research, Industrial Marketing Management y Marketing Science. Se revisaron un total de 151 artículos de investigación que trataron aspectos relacionados al uso de la Gestión de Relaciones con Clientes (CRM) durante la década 2010-2019. Se realizó la clasificación del total de artículos en base a los siguientes criterios: por año de publicación, por tipo de investigación (empírica-teórica), según el tamaño de la empresa, por temas clave de cada artículo que incluye la presentación de referencias utilizadas en cada temática identificada. Se incluyeron solo artículos de referencia excluyendo libros o ponencias.

El procedimiento se efectuó con rigurosidad y orden a través de cuatro etapas. En la primera fase se clasificó los artículos de CRM por año de publicación con el objetivo de conocer la mayor y menor producción de investigaciones de CRM en cada revista. Luego se realizó una codificación de los artículos en base al tipo de investigación utilizado ya sea teórico o empírico recordando que el objetivo del presente trabajo es conocer la evolución de investigaciones empíricas relacionadas a prácticas de CRM en pequeñas empresas.

En una segunda etapa se clasificó a los artículos que desarrollaron investigación según el tamaño de empresa considerando para ello seis subcategorías: pequeña empresa; mediana empresa; gran empresa; pequeña-mediana empresa; pequeña, mediana y gran empresa y; finalmente, se identificó estudios concentrados en la mediana y gran empresa.

En la tercera etapa se procedió a clasificar las investigaciones por temas claves de CRM, 
según el número de artículos. En este apartado se concluyó con el reporte de referencias revisadas, en función a cada temática identificada.

Toda la información se registró en una matriz general y cuadros de doble entrada.

\section{RESULTADOS}

En las siguientes líneas se presenta los resultados según los criterios de clasificación indicados, considerando en primer lugar el número de artículos por año de publicación.

La producción científica de artículos de CRM por año de publicación durante la década 2010-2019 alcanzó un máximo de 22 investigaciones el 2010 y un mínimo de 9 trabajos publicados el 2012. Asimismo, en promedio se publicó 15 artículos de CRM en las 5 principales revistas de marketing durante los 10 años analizados.

Un dato importante es que en los primeros cinco años se logró la mayor producción de artículos de CRM alcanzando 81 investigaciones que representa el $53.3 \%$ del total; en contraste con el segundo quinquenio que reporta 70 artículos representado por el $46.2 \%$, según la Figura 1, que a su vez muestra una tendencia homogénea de producción de artículos en los últimos tres años.
Asimismo, como información adicional se destaca que la revista de marketing que logró mayor publicación de artículos de CRM en la última década fue el Journal of the Academy Marketing Science caracterizado por una producción ininterrumpida de publicaciones alcanzando 71 artículos de CRM; por su parte, la revista con menor producción y con periodos anuales vacíos fue Journal of Marketing Research que a lo largo de la década analizada solo publicó 10 artículos.

A su vez, se presenta los resultados teniendo en consideración la clasificación de artículos según el tipo de investigación: empírica o teórica. Al respecto, de los 151 artículos de CRM se identificó 125 artículos (83\%) correspondientes a investigaciones de tipo empírico los cuales utilizaron encuestas y fichas de observación para la recopilación de datos y el trabajo de campo y; por otro lado, se halló y revisó 26 artículos (17\%) considerados en el perfil de trabajos teóricos; los cuales incluyeron investigaciones de revisión de la literatura.

Una siguiente clasificación de los artículos se ordenó según el tamaño de empresa donde se aplicó el estudio. En tal sentido, se detectó que la mayoría de investigaciones de CRM registra un permanente interés en conseguir información de grandes corporaciones e industrias. Como se

Figura 1

Distribución de artículos de CRM por año de publicación, periodo 2010-2019

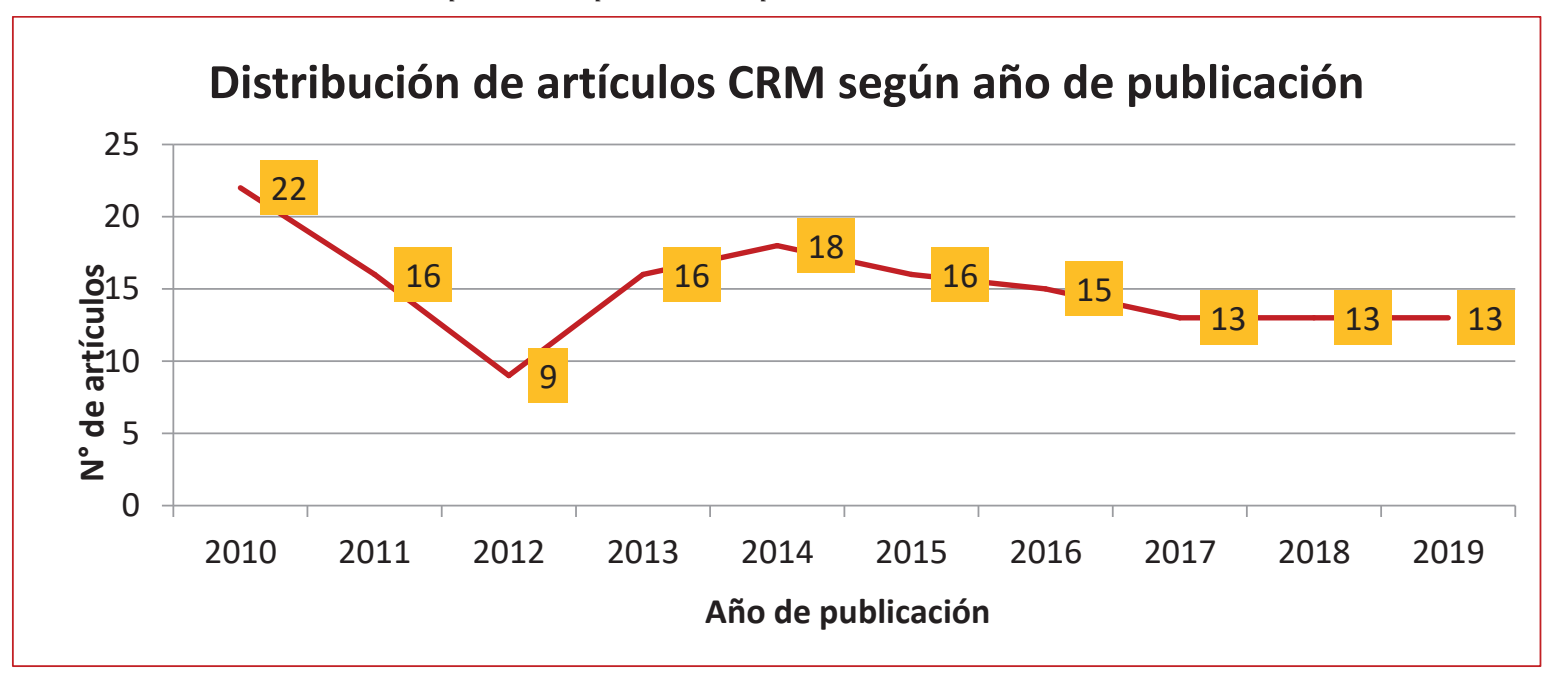

Nota. Elaboración propia con información de las cinco mejores revistas de Marketing ubicadas en el primer cuartil Q1, según SCImago Journal Rank. 
muestra en la Figura 2, un total de 72 artículos empíricos de CRM se concentra en la gran empresa, las cuales se caracterizan por tener estructuras funcionales fluidas y alta capacidad de inversión en capital humano y económico. Seguido de 18 artículos con datos de la gran y mediana empresa; a su vez, un total de 12 artículos obtuvieron información de la pequeña, mediana y gran empresa. Por otro lado, 10 investigaciones se focalizaron en la realidad de la mediana empresa, seguido de 7 artículos dirigido a la pequeña empresa. El menor interés, con 6 publicaciones, está en artículos comparativos que extrajeron datos simultáneamente tanto de la pequeña como de la mediana empresa.

Asimismo, es importante destacar que en la realidad analizada se evidencia limitados estudios de CRM en la pequeña empresa tanto a nivel del sector comercial y de servicios; éste último de exponencial crecimiento en la era digital como los casos de experiencias de CRM en empresas online. Se detectó también el manejo de información empírica híbrida; es decir, comparaciones en un mismo estudio según el tamaño de la organización. La primera de ellas es el interés por recolectar y comparar información empírica entre la pequeña y mediana empresa; luego entre la pequeña, mediana y gran empresa; y finalmente, la mediana y gran empresa.

Una última clasificación de los artículos revisados fue en base a los temas de CRM de mayor y menor interés. El "impacto de programas CRM" y "estrategias CRM y rentabilidad" fueron los dos ejes temáticos más investigadas sumando un total de 72 artículos; en comparación a los temas menos abordados "comportamiento del consumidor" y "antecedentes de CRM" que suman 15 artículos en total. Se evidencia el gran interés de la comunidad científica por conocer cuáles son "los factores de éxito y causas de fracaso en la implementación de CRM" (Garrido y Padilla, 2011, p. 49).

Al respecto, los estudios numerosos de Fletcher y Wright (1996), Perrien et al. (1993), citado en Ryals y Knox (2001) concluyen que la implementación exitosa de todo programa de CRM debe tener en cuenta factores mediadores como "el compromiso total de la alta dirección por trabajar una cultura organizativa centrada en el cliente y la gestión eficaz de áreas interfuncionales implicadas de la empresa" (p. 540).

Los trabajos de investigación de Zablah, Bellenger y Johnston (2004) en importantes

Figura 2

Clasificación de artículos CRM, según tamaño de la empresa, periodo 2010-2019

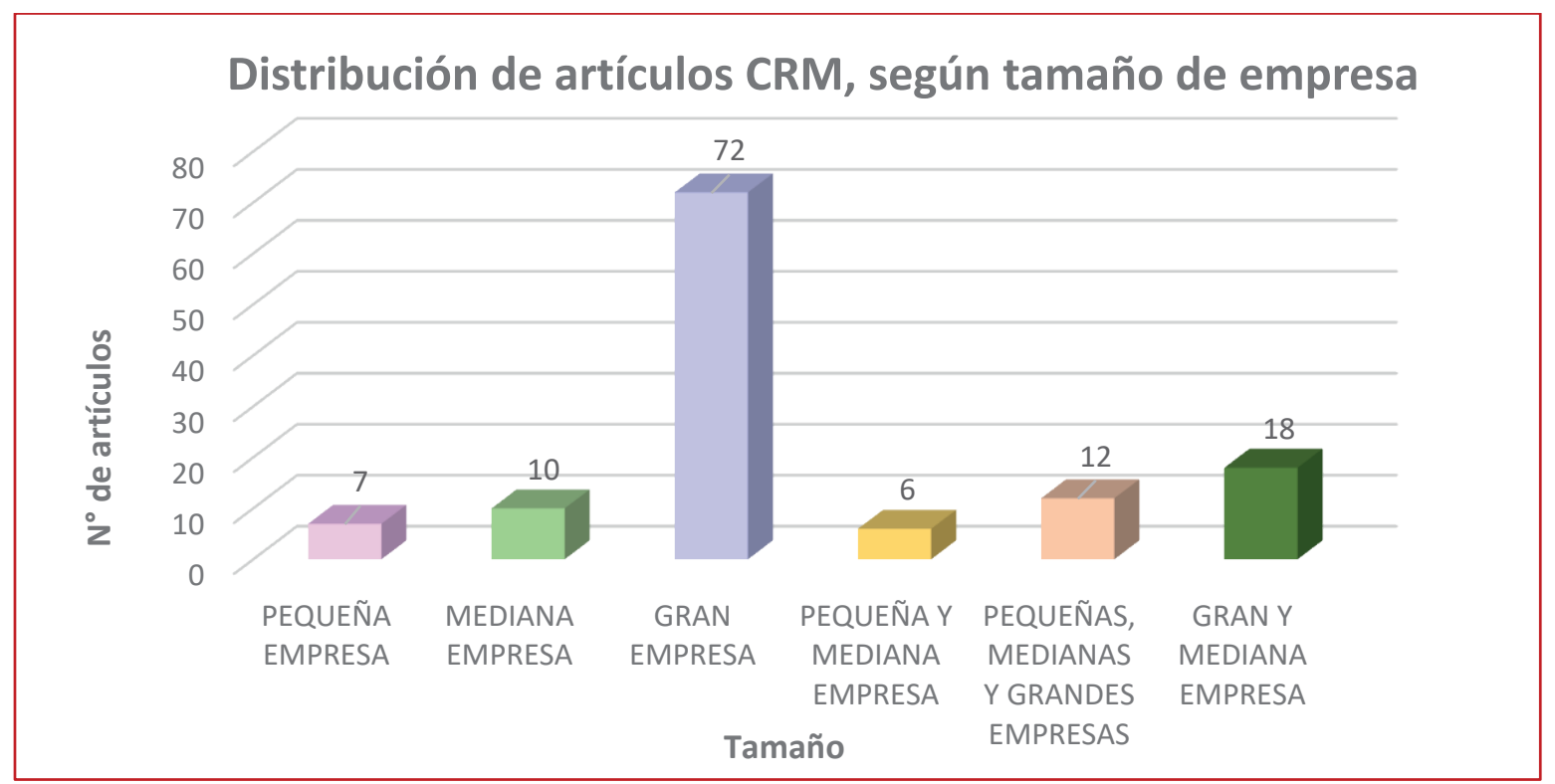

Nota. Elaboración propia con información de las cinco mejores revistas de Marketing ubicadas en el primer cuartil Q1, según SCImago Journal Rank. 
industrias americanas y europeas proponen una serie de fases para el éxito de iniciativas CRM. "Se destaca la estrategia a seguir, el determinar roles y procesos CRM, evaluar las capacidades de gestión de conocimiento y de interacciones y finalmente, monitorear para mejorar los procesos" (Zablah, Bellenger y Johnston, 2004, p. 485).

Asimismo, las áreas temáticas de un progresivo interés y crecimiento en el tiempo se focalizaron en aspectos relacionados al valor de vida del cliente (21 artículos) y al e-CRM que incluye redes sociales y tecnología CRM así como herramientas de gestión de datos llámese Data Mining y Big Data (43 artículos), según indica la Figura 3. En un contexto digital como el actual esta lista de temas despierta el interés científico al explicar el comportamiento de grandes datos ya que permiten optimizar los procesos de atención al cliente en la gran industria que invierte dinero en el acceso a redes y tecnología de punta.

Por otro lado, los temas relacionados al "comportamiento del consumidor" así como la "definición y antecedentes del CRM" obtuvieron un mínimo de interés en el total de artículos revisados alcanzando un índice porcentual bajo de $4.5 \%$ y $5.2 \%$, (7 y 8 artículos) respectivamente. Los resultados hallados son muy similares al estudio de Sota et al. (2018) que realizó una revisión de prácticas de CRM del 2007 al 2016 descubriendo que uno de los comportamientos del consumidor global es que responde favorablemente a "estrategias de marketing internacional, según su nacionalidad y perspectiva cultural, en un entorno cada vez más digital e intercultural apelando el estudio en mención a que los especialistas de marketing deben considerar estos aspectos al evaluar programas de lealtad" (p. 5). Ver Figura 3.

A continuación, la Tabla 1 proporciona una extensa lista de referencias de la literatura de CRM revisada en orden cronológico descendente (2019-2010), según los temas clave de mayor y menor interés en investigaciones empíricas de CRM, a fin de tener un panorama completo de autores especializados en diversos tópicos inmersos en CRM. Ello permitirá a futuros in-

\section{Figura 3}

Clasificación de artículos CRM, según área temática, periodo 2010-2019

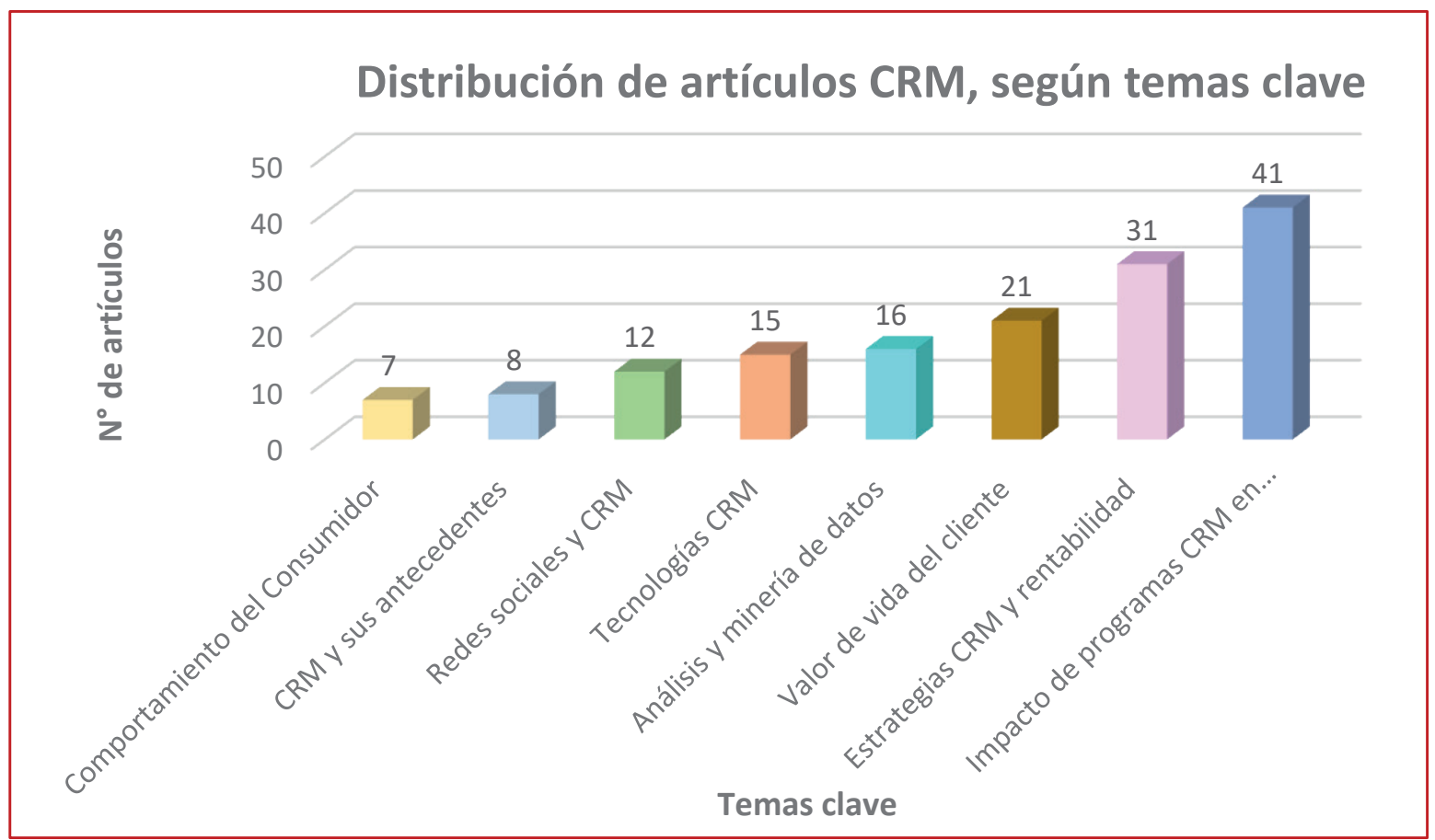

Nota. Elaboración propia con información de las cinco mejores revistas de Marketing ubicadas en el primer cuartil Q1, según SCImago Journal Rank. 
Tabla 1

Referencias de artículos revisados y clasificados por temas de CRM, periodo 2010-2019.

\begin{tabular}{ll}
\hline TEMAS CLAVE & BIBLIOGRAFÍA REVISADA 2010-2019 \\
\hline Comportamiento del & Kumar, V., Leszkiewicz, A. y Herbst, A. (2018); Kähr, A., Nyffenegger, B., Krohmer, H. y Hoyer, WD (2016); Leach, \\
Consumidor & MPy Liu, AH (2014); Guercini, S., La Rocca, A., Runfola, A. y Snehota, I. (2014); Ascarza, E. y Hardie, BGS (2013); \\
& Menguc, B., Auh, S. y Uslu, A. (2012); Shin, J. y Sudhir, K. (2010).
\end{tabular}

CRM y sus antecedentes

Redes sociales y CRM

Tecnologías CRM

Análisis y minería de datos

Valor de vida del cliente

Estrategias CRM y rentabilidad

Impacto de programas CRM en la Fidelización o Lealtad

Hartmann, NN, Wieland, H. y Vargo, SL (2018); Moorman, C. y Day, GS (2016); Yao Zhang, Eric T. Bradlow y Dylan S. Small. (2015); Cron, WL, Baldauf, A., Leigh, TW y Grossenbacher, S. (2014); Jelinek, R. (2017); Rust, RT y Huang, M.-H. (2014); Makkonen, H. y Olkkonen, R. (2013); Kalaignanam, K. y Varadarajan, R. (2011).

Ancillai, C., Terho, H., Cardinali, S. y Pascucci, F. (2019); Vieira, VA, de Almeida, MIS, Agnihotri, R., da Silva, NSDAC y Arunachalam, S. (2019); Indmarman, J. (2019); Ogilvie, J., Agnihotri, R., Rapp, A. y Trainor, K. (2018); Nunan, D., Sibai, O., Schivinski, B. y Christodoulides, G. (2018); Itani, OS, Agnihotri, R. y Dingus, R. (2017); Kumar, A., Bezawada, R., Rishika, R., Janakiraman, R. y Kannan, PK (2016); Järvinen, J. y Karjaluoto, H. (2015); Järvinen, J. y Taiminen, H. (2016); Wang, WYC, Pauleen, DJ y Zhang, T. (2016); Ma, L., Sun, B. y Kekre, S. (2015); Mitrega, M., Forkmann, S., Ramos, C. y Henneberg, SC (2012).

Pagani, M. y Pardo, C. (2017); Huang, M.-H. y Rust, RT (2017); Hunter, GK y Panagopoulos, NG (2015); Limbu, YB, Jayachandran, C. y Babin, BJ (2014); Obal, M. (2013); Obal, M. y Lancioni, RA (2013); Mariadoss, BJ, Milewicz, C., Lee, S. y Sahaym, A. (2014); Hakanen, T. (2014); Davies, IA y Ryals, LJ (2014); Kumar, V., Dixit, A., Javalgi, RG y Dass, M. (2015); Lee, RP, Johnson, JLy Tang, X. (2012); Trainor, KJ, Rapp, A., Beitelspacher, LS y Schillewaert, N. (2011); Ahearne, M., Lam, SK, Mathieu, JE y Bolander, W. (2010); Kim, N., Pae, JH, Han, JK y Srivastava, RK (2010); Mitręga, M. y Katrichis, JM (2010).

Venkatesan, R., Bleier, A., Reinartz, W. y Ravishanker, N. (2019); Zhang, JZ (2019); Kouropalatis, Y., Giudici, A. y Acar, OA (2018); Dew, R. y Ansari, A. (2018); Wedel, M. y Kannan, PK (2016); Bone, SA, Lemon, KN, Voorhees, CM, Liljenquist, KA, Fombelle, PW, Detienne, KB y Money, RB (2017); Guercini, S., La Rocca, A., Runfola, A. y Snehota, I. (2015); Thompson, SA, Gooner, RA y Kim, A. (2014); Tamaddoni Jahromi, A., Stakhovych, S. y Ewing, M. (2014); Stein, AD, Smith, MF y Lancioni, RA (2013); Ling-yee, L. (2011); Alshawi, S., Missi, F. e Irani, Z. (2011); Salojärvi, H., Sainio, L.-M. y Tarkiainen, A. (2010); Korhonen-Sande, S. (2010); Lorentz, H. y Ghauri, PN (2010); Madhavaram, S. y McDonald, RE (2010).

Stahl, F., Heitmann, M., Lehmann, DR y Neslin, SA (2012); Kumar, V. y Reinartz, W. (2016); Neslin, SA, Taylor, GA, Grantham, KD y McNeil, KR (2012); Hanson, S. y Yuan, H. (2017); Sunder, S., Kumar, V. y Zhao, Y. (2016); Balboni, B. y Terho, H. (2016); Arslanagic-Kalajdzic, M. y Zabkar, V. (2015); Töytäri, P. y Rajala, R. (2015); Esteban-Bravo, M., Vidal-Sanz, JM y Yildirim, G. (2014); Hunter, GK (2014); ; Corsaro, D. (2014); Kim, D., Cavusgil, ST y Cavusgil, E. (2013); Keränen, J. y Jalkala, A. (2013); Jaakkola Jaakkola, E. y Hakanen, T. (2013); Keränen, J. y Jalkala, A. (2013); Krush, MT, Agnihotri, R., Trainor, KJ y Nowlin, EL (2013); Chan, TY, Wu, C. y Xie, Y. (2011); Holm, M., Kumar, V. y Rohde, C. (2011); Sun, B. y Li, S. (2011); Blocker, CP, Flint, DJ, Myers, MB y Slater, SF (2010); Melancon, JP, Noble, SM y Noble, CH (2010).

Dose, DB, Walsh, G., Beatty, SE y Elsner, R. (2019); Gupta, A., Kumar, A., Grewal, R. y Lilien, GL (2019); Koponen, J., Julkunen, S. y Asai, A. (2019); Kim, M., Sudhir, K., Uetake, K. y Canales, R. (2019); Kanuri, VK y Andrews, M. (2019); Miller, CJ, Wiles, MA y Park, S. (2019); Kim, KH y Kumar, V. (2018); Kumar, V., Lahiri, A. y Dogan, OB (2018); Dawson, B., Young, L., Murray, JM y Wilkinson, I. (2017); Johnson, JS, Matthes, JM y Friend, SB (2017); Terho, H. y Jalkala, A. (2017); Limon, KN y Verhoef, PC (2016); Virtanen, T., Parvinen, P. y Rollins, M. (2015); Möller, K. y Parvinen, P. (2015); Wang, XL y Brennan, R. (2014); Bonney, L., Plouffe, CR y Wolter, J. (2014); D'Haen, J. y Van den Poel, D. (2013); Stanko, MA, Bohlmann, JD y Molina-Castillo, F.-J. (2013); Steel, M., Dubelaar, C. y Ewing, M. T. (2013); Homburg, C., Bornemann, T. y Kretzer, M. (2013); Rollins, M., Bellenger, DN y Johnston, WJ (2012); Schmitz, C. (2012); Iyengar, R., Jedidi, K., Essegaier, S. y Danaher, PJ (2011); Avlonitis, GJ y Panagopoulos, NG (2010); Davies, IA, Ryals, LJ y Holt, S. (2010); Viswanathan, M., Rosa, JA y Ruth, JA (2010); Keramati, A., Mehrabi, H. y Mojir, N. (2010); Ernst, H., Hoyer, WD, Krafft, M. y Krieger, K. (2010); Park, JE, Kim, J., Dubinsky, AJ y Lee, H. (2010); Lim, SC y Lusch, RF (2010); Vorhies, DW, Orr, LM y Bush, VD (2010).

Chaudhuri, M., Voorhees, CM y Beck, JM (2019); Lawrence, JM, Crecelius, AT, Scheer, LK y Lam, SK (2019); Ascarza, E. (2018); Newman, CL, Cinelli, MD, Vorhies, D. y Folse, JAG (2018); Wang, YJ, Capon, N., Wang, VL y Guo, C. (2018); Kumar, V., Nim, N. y Sharma, A. (2018); Crecelius, AT, Lawrence, JM, Lee, J.-Y., Lam, SK y Scheer, LK (2018); Saboo, AR, Kumar, V. y Anand, A. (2017); Woisetschläger, DM, Backhaus, C. y Cornwell, TB (2017); Kumar, V., Rajan, B., Gupta, S. y Pozza, ID (2017); Hanson, S. y Yuan, H. (2017); Ascarza, E., Ebbes, P., Netzer, O. y Danielson, M. (2017); Ou, Y.-C., Verhoef, PC y Wiesel, T. (2016); Hollebeek, LD, Srivastava, RK y Chen, T. (2016); Pansari, A. y Kumar, V. (2016); Min, S., Zhang, X., Kim, N. y Srivastava, RK (2016); Zhang, J., Jiang, Y., Shabbir, R. y Du, M. (2015); Homburg, C., Jozić, D. y Kuehnl, C. (2015); Pick, D., Thomas, JS, Tillmanns, S. y Krafft, M. (2015); Lee, J.-Y., Sridhar, S., Henderson, CM y Palmatier, RW (2015); Verma, V., Sharma, D. y Sheth, J. (2015); DeCarlo, TE y Lam, SK (2015); Steinhoff, L. y Palmatier, RW (2014); Mullins, RR, Ahearne, M., Lam, SK, Hall, ZR y Boichuk, JP (2014); ALHussan, FB, AL-Husan, FB y Fletcher-Chen, CC-Y. (2014); Huikkola, T., Ylimäki, J. y Kohtamäki, M. (2013); Ekici, A. (2013); Johnson, DS, Clark, BH y Barczak, G. (2012); Enz, MG y Lambert, DM (2012); Shi, G., Shi, Y., Chan, AKK, Liu, MT y Fam, K.-S. (2011); Chen, J.-S., Tsou, H.-T. y Ching, RKH (2011); Williams, P., Khan, MS, Ashill, Nueva Jersey y Naumann, E. (2011); Hillebrand, B., Nijholt, JJ y Nijssen, EJ (2011); Luo, X., Wieseke, J. y Homburg, C. (2011); Jing, X. y Lewis, M. (2011); Williams, P., Khan, MS, Ashill, Nueva Jersey y Naumann, E. (2011); Ballantyne, D., Frow, P., Varey, RJ y Payne, A. (2011); Fock, H., Yim, F. y Rodríguez, M. (2010); Arnold, TJ, (Er) Fang, E. y Palmatier, RW (2010); Homburg, C., Müller, M. y Klarmann, M. (2010); Briggs, E., Landry, TD y Daugherty, PJ (2010).

Nota. Elaboración propia con información de las cinco mejores revistas de Marketing ubicadas en el primer cuartil Q1, según SCImago Journal Rank. 
vestigadores especializados en marketing relacional continuar profundizando diversos temas y enfoques de CRM para detectar nuevas tendencias en los próximos diez años.

Se observa en la tabla 1 cómo la mayor parte de la bibliografía analizada se concentra en temas que tratan el impacto de programas y uso de estrategias CRM. Asimismo, al revisar los subtemas clave se detectó que estudios relacionados a factores de éxito y causas del fracaso en la implementación CRM recibieron la mayor atención de investigadores como Ballantyne, D., Frow, P., Varey, RJ y Payne, A. (2011); Fock, H., Yim, F. y Rodríguez, M. (2010); Chaudhuri, M., Voorhees, CM y Beck, JM (2019); Lawrence, JM, Crecelius, AT, Scheer, LK y Lam, SK (2019); Ascarza, E. (2018); Newman, CL, Cinelli, MD, Vorhies, D. y Folse, JAG (2018, entre otros, según se observa en la Tabla 1).

\section{DISCUSIÓN}

A la luz del objetivo trazado en el presente estudio que fue identificar el nivel de interés por investigaciones en el ámbito de la Gestión de Relaciones con Clientes (CRM) en pequeñas empresas; los resultados obtenidos muestran que existe una escasa revisión de literatura académica dirigido a pequeños negocios. De 151 artículos revisados que tratan del modelo de administración denominado Gestión de Relaciones con Clientes (CRM) en las principales cinco revistas de marketing de mayor impacto mundial, en el periodo 2010-2019, se halló que 125 son publicaciones de carácter empírico y 26 teóricos. Por lo tanto, del total de investigaciones empíricas, solo 7 artículos de CRM se enfocan en conocer la realidad de la pequeña empresa, en contraste con 72 publicaciones que registran gran interés científico en profundizar diversas temáticas de CRM en la gran empresa.

Es evidente una amplia literatura de CRM inclinado a la gran empresa donde destacan industrias y trasnacionales del sector de telecomunicaciones, automotriz y farmacia. Esta tendencia de otorgar importancia significativa a la gran industria no está permitiendo conocer a directivos y a la academia información referente a cuáles son las estrategias CRM más efectivas para fidelizar clientes y saber qué factores determina una implementación exitosa del CRM en las pequeñas empresas que se ubican en provincias y distritos de la periferia en las grandes capitales.

En lo referente al interés por investigaciones empíricas, los resultados hallados se relacionan con los obtenidos por Sota S., Chaudhry H., Chamaria A. y Chauhan A. (2018), quienes luego de revisar estudios de CRM en la década 2007-2016 detectaron la existencia de una alta concentración de artículos de CRM de tipo empírico a través de encuestas señalando que el $86 \%$ de la literatura de CRM son empíricas y el $14 \%$ restante fueron artículos de investigación conceptual. Sin embargo, no se halló coincidencias de resultados de estudios de CRM según el tamaño de empresa porque no fue criterio de interés en la investigación de Sota S. y colaboradores.

A su vez, los resultados de esta investigación respecto al tema de mayor interés en la literatura CRM en el periodo 2010-2019 es el impacto de programas CRM en la organización, concuerdan con los de Garrido A. y Padilla A. (2011), quienes señalan que uno de los factores de éxito para un impacto favorable del CRM es la existencia de una cultura corporativa centrada en el cliente, el compromiso de los ejecutivos y contar con los recursos tecnológicos necesarios así como colaboradores capacitados para llevar adelante programas y estrategias CRM.

\section{CONCLUSIONES}

1. En la última década las revistas de marketing de mayor impacto a nivel mundial presentan un escaso interés por investigar prácticas de Gestión de Relaciones con Clientes (CRM) en pequeñas empresas.

2. Hay un creciente interés y alta producción científica de trabajos empíricos de temas relacionados a prácticas de CRM enfocados en la gran empresa, específicamente en el sector industrial que incluye organizaciones trasnacionales del sector telecomunicaciones, automotriz y farmacia.

3. Los subtemas de CRM de mayor interés en la década analizada son los relacionados a tecnología, minería de datos aplicados al consumidor y programas 
de implementación de CRM para la fidelización de clientes.

4. En el primer quinquenio hubo una limitada producción de artículos de CRM en la mayoría de las revistas analizadas, en comparación a los últimos 5 años donde se estabilizó la producción a un promedio de 14 artículos por año; lo que demuestra que en los últimos años la estrategia CRM está siendo revalorada en las empresas y en los centros de investigación.

5. El principal aporte del presente estudio al acervo científico es que presenta un reporte cuantitativo de los últimos diez años sobre prácticas de CRM en empresas a fin de reflexionar y equilibrar en el futuro los estudios hacia otras realidades organizacionales como las pequeñas empresas, las cuales cada vez más ejercen un rol importante en los mercados y en las economías emergentes.

\section{REFERENCIAS BIBLIOGRÁFICAS}

Dalla Pozza, I., Goetz, O. y Sahut, JM (2018). Efectos de la implementación en la relación entre CRM y su desempeño. Journal of Business Research, 89 (2018), 391-403. https://doi.org/10.1016/j. jbusres.2018.02.004.

Finnegan, D. y Currie, W. (2010). Un enfoque de múltiples capas para la implementación de CRM: una perspectiva de integración. European Management Journal, 28 (2), 153-167. https://doi. org/10.1016/j.emj.2009.04.010.

Garrido, A. y Padilla, A. (2011). El CRM como estrategia de negocio: desarrollo de un modelo de éxito y análisis empírico en el sector hotelero español. Revista Europea de Dirección y Economía de la Empresa, 20 (2), 101-118. https://doi. org/10.1016/j.ijinfomgt.2011.01.002

Ngai, E. (2005). Customer Relationship Management Research 1992-2002. An Academic Literatura review and classification. Marketing Intelligence \& Planning, 23 (6), 582-605. https:// doi.org/10.1108/02634500510624147.
Nguyen, B. y Mutum, DS. (2012). Gestión de Relaciones con Clientes: avances, lados oscuros, explotación e injusticia. Gestión electrónica de relaciones con el cliente, 6(1), 1-19. https://doi. org/10.1108/14637151211232614.

Pan, S., \& Lee, J. (2003). Using e-CRM for a unified view of the customer. Communications of the ACM. Communications oj the ACM, 46(4), 95-99. https://doi.org/10.1145/641205.641212

Ryals, L.; Knox, S. (2001). Problemas multifuncionales en la implementación del marketing relacional a través de la gestión de relaciones con el cliente. European Management Journal, 19(5), 534-542. https://doi.org/10.1016/S02632373(01)00067-6

Sota S., Chaudhry H., Chamaria A. y Chauhan A. (2018). Investigación sobre Gestión de Relaciones con los Clientes de 2007 a 2016: Revisión de literatura académica. Revista de Marketing Relacional. 0 (0), 1-15. https://doi.org/10.1080 /15332667.2018.1440148.

Wahlberg, O., Strandberg,C., Sundberg, H. y Sandberg, K. (2009).Tendencias, temas y áreas poco investigadas en la investigación de CRM: una revisión de la literatura. International Journal of Public Information Systems, 3 (0), 191-208. http://urn.kb.se/resolve?urn=urn:nbn:se:du-15394</div $></$ div $>$

Zablah A., Bellenger D. y Johnston (2004). Una evaluación de perspectivas divergentes sobre la relación con el cliente. Hacia un entendimiento común de un fenómeno emergente. Gestión de Marketing Industrial, 33 (2004), 475-489. https://doi.org/10.1016/j.indmarman.2004.01.006

Zikmund, W., McLeod, R. y Gilbert, F. (2004). Administración de relaciones con los clientes. México: Compañía Editorial Continente. 
